\section{Grossesse extra-utérine}

\section{Extra-uterine pregnancy}

\section{A. Hutin · J. Mayer - A. Santin}

Reçu le 17 août 2013; accepté le 5 octobre 2013

(C) SFMU et Springer-Verlag France 2013

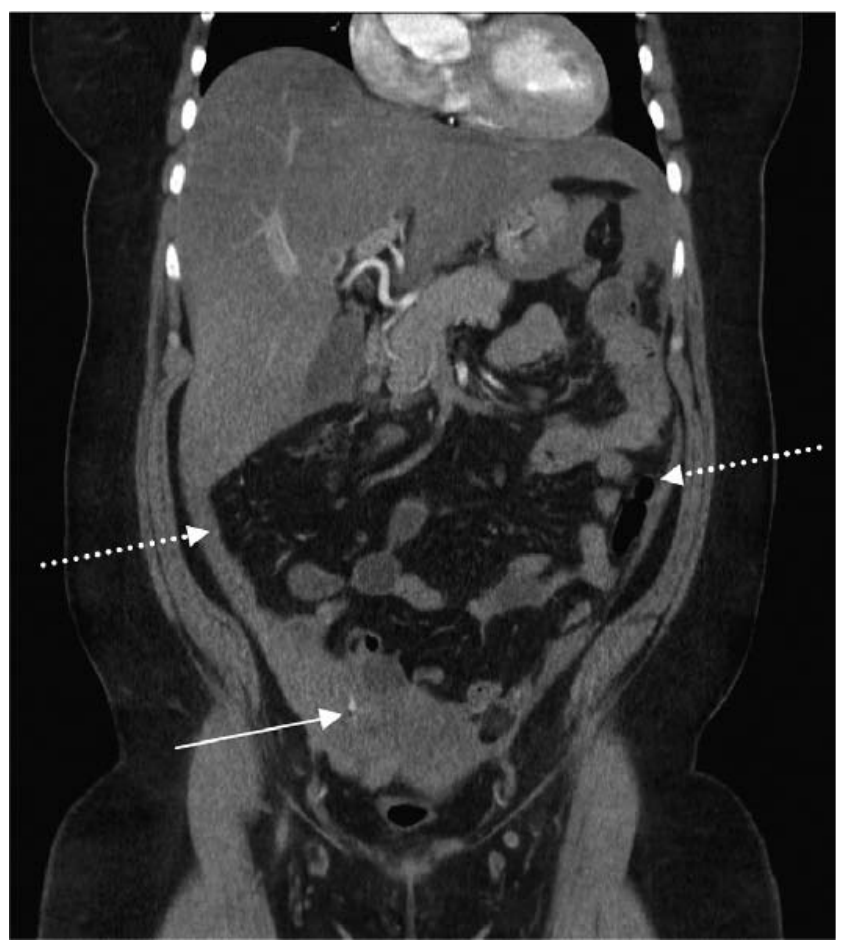

Fig. 1 TDM abdomino-pelvien injecté. Coupe tomodensitométrique coronale, temps artériel

Flèches pointillées : hémopéritoine. Flèche pleine : saignement actif latéro-utérin droit

\footnotetext{
A. Hutin $(\bowtie)$

Service des urgences, CHU Henri Mondor,

Assistance publique-hôpitaux de Paris,

université Paris Est Créteil, 51, avenue du Maréchal Delattre

de Tassigny, F-94000 Créteil, France

e-mail : alice.hutin@hmn.aphp.fr

\section{J. Mayer}

Service de radiologie, CHU Henri Mondor,

Assistance publique-hôpitaux de Paris,

université Paris Est Créteil, 51, avenue du Maréchal Delattre

de Tassigny, F-94000 Créteil, France

\section{A. Santin}

Service des urgences, Hôpitaux Universitaires Paris Centre, Assistance publique-hôpitaux de Paris, F-75014 Paris, France
}

Une patiente de 45 ans consulte aux urgences pour des douleurs abdominales d'apparition brutale, sans fièvre associée. Les douleurs ont débuté en région pelvienne puis migré secondairement en regard de l'hypochondre droit. À son arrivée, la patiente présente une instabilité hémodynamique associée à une défense du flanc et de l'hypochondre droits. Après stabilisation de l'état hémodynamique, une tomodensitométrie abdominopelvienne est réalisée à visée diagnostique. Cet examen met en évidence un hémopéritoine remontant jusqu'au niveau du foie et de la rate (Fig. 1) (flèches pointillées), avec un saignement actif latéro-utérin droit (Fig. 1)(flèche pleine : image hyperdense au temps injecté) faisant alors suspecter un kyste ovarien hémorragique ou une grossesse extra-utérine (GEU). Le taux de béta-HCG (9518 UI/l) prélevé à l'arrivée de la patiente confirme secondairement le diagnostic de GEU rompue. La patiente bénéficie d'une transfusion de deux culots globulaires associée à un traitement chirurgical en urgence. 